\title{
Quantitative Analysis of Glycine Oligomerization by Ion-Pair Chromatography
}

Thomas D. Campbell, ${ }^{1}$ Rio Febrian, ${ }^{1}$ Holly E. Kleinschmidt, ${ }^{1}$ Kevin A. Smith, ${ }^{1}$ and Paul J. Bracher ${ }^{1, *}$

${ }^{1}$ Department of Chemistry, Saint Louis University, 3501 Laclede Avenue, St. Louis, Missouri 63103 U.S.A.

* Corresponding Author. E-mail: paul.bracher@slu.edu

Submitted for Publication in

ACS Omega 


\section{Contents}

Supplemental Experimental

General Considerations

Quantitative NMR Spectroscopy

UV-vis Spectroscopy

Estimation of Detectable Oligomers

Statistical Treatment of Uncertainty and Error

Analysis of the Gly 8 Standard

Equations for extinction coefficients (S1) and hypothesis testing (S2) S6

Practical Notes

Method Development

$\mathrm{pH}$ of Mobile Phase

Limits of Quantitation

Control Experiment for Interference of DKP

Figure S1. IP-HPLC chromatogram of Gly8 $\quad$ S9

Figure S2. Example measurements of Gly4 standards using IP-HPLC $\quad$ S10

Figure S3. Calibration curve for Glycine $\quad$ S11

Figure S4. Calibration curve for Gly2 $\quad$ S11

Figure S5. Calibration curve for $\mathrm{Gly}_{3} \quad \mathrm{~S} 12$

$\begin{array}{ll}\text { Figure S6. Calibration curve for } \mathrm{Gly}_{4} & \mathrm{~S} 12\end{array}$

$\begin{array}{ll}\text { Figure S7. Calibration curve for } \mathrm{Gly}_{5} & \text { S13 }\end{array}$

$\begin{array}{ll}\text { Figure S8. Calibration curve for } \mathrm{Gly}_{6} & \text { S13 }\end{array}$

Figure S9. Calibration curve for Gly8 $\quad$ S14

Figure S10. Chromatogram of mixed standard of Gly $\mathrm{y}_{1-6} \quad \mathrm{~S} 15$

Table S1. Approximate retention times of $\mathrm{Gly}_{\mathrm{n}}$ standards $\quad$ S15

Figure S11. NMR spectrum of standard solution of Glycine $\quad$ S16

Figure S12. NMR spectrum of standard solution of $\mathrm{Gly}_{2} \quad$ S16

$\begin{array}{ll}\text { Figure S13. NMR spectrum of standard solution of } \mathrm{Gly}_{3} & \text { S17 }\end{array}$

$\begin{array}{ll}\text { Figure S14. NMR spectrum of standard solution of } \mathrm{Gly}_{4} & \text { S17 }\end{array}$

$\begin{array}{ll}\text { Figure S15. NMR spectrum of standard solution of } \mathrm{Gly}_{5} & \text { S18 }\end{array}$

$\begin{array}{ll}\text { Figure S16. NMR spectrum of standard solution of Gly } 6 & \text { S18 }\end{array}$

Figure S17. NMR spectrum of mixed standards of Gly $1-6 \quad$ S19

Figure S18. UV-vis spectrum of a standard of Gly4 and a blank spectrum of the IP reagent $\quad$ S20

Table S2. Measured UV responses for a mixed standard of Gly $(n=1-6) \quad$ S21

Table S3. Measured UV responses for Gly $y_{n}$ products from an oligomerization reaction of glycine $\quad$ S21

Figure S19. IP-HPLC chromatogram of low-molecular-weight polyglycine $\quad$ S22

Table S4. Measured limits of quantitation (LOQ) for Gly through Gly $_{6} \quad$ S23

$\begin{array}{ll}\text { Supplemental References } & \text { S24 }\end{array}$ 


\section{Supplemental Experimental}

General Considerations. All reagents, including glycine, oligomers of glycine up to Gly6, and low-molecular-weight (LMW) polyglycine were obtained from MilliporeSigma or VWR and their affiliate suppliers and were not purified further. A standard of Gly8 was ordered for custom synthesis from Fisher Scientific USA. The purity of this sample was checked by IP-HPLC and found to be contaminated by other oligomers of glycine, including Gly, Gly6, Gly7, and Gly9 (Figure S1). As a result, we did not incorporate data collected on this sample of Glys into our model for the extrapolation of molar response factors $\left(f_{\mathrm{n}}\right)$ of higher oligomers.

Standard solutions of each oligomer were prepared using a minimum of $10 \mathrm{mg}$ of sample, and the solutions were prepared using a volumetric flask (of at least $50 \mathrm{~mL}$ ) for accuracy. These solutions were diluted to five different concentrations using micropipettes, giving five individual standards for each oligomer (Figure S2). This procedure was repeated three different times for each oligomer of glycine, yielding three measurements for each oligomer at five different concentrations (Figures S3-S9). The three trials for each oligomer at each concentration were run with unique samples that were prepared individually for the determination of the molar response factor $\left(f_{\mathrm{n}}\right)$.

Quantitative Nuclear Magnetic Resonance (qNMR) Spectroscopy. The exact concentration of each standard was verified using quantitative ${ }^{1} \mathrm{H}$ NMR spectroscopy by comparing the signal from the analyte to that of an internal standard of $2.5 \mathrm{mM}$ tert-butanol in $\mathrm{D}_{2} \mathrm{O}$ (Figures S11-S16). All spectra were collected using a solvent-suppression, 1-D excitation sculpting pulse program ('zgesgp' on Bruker's TopSpin 3.2 software). To ensure quantitative integration of the signals, the recycle delay (D1) was set to 15 seconds, which is approximately

seven times the spin-lattice relaxation $\left(T_{1}\right)$ for a glycine oligomer. ${ }^{1-2}$ The low solubility of Gly6 in 
water resulted in an NMR spectrum of poor quality. Despite the poor quality of this spectrum, the concentration of the Gly6 standard determined by qNMR spectroscopy closely matched the target concentration based on the mass of the analyte and the volume of the standard solution.

UV-Vis Spectroscopy. We collected a UV-vis spectrum of a Gly4 standard in the IPHPLC mobile phase solvent to help determine wavelengths for detection in the method. We selected $195 \mathrm{~nm}$ to match previous reports and because there was no obvious better alternative (see Figure S18). We also had the detector observe $214 \mathrm{~nm}$, which is a commonly used wavelength for the analysis of peptides. The sensitivity at $214 \mathrm{~nm}$ is considerably lower than 195 $\mathrm{nm}$ and may not be suitable for analyzing higher oligomers that are likely to be present in low concentrations due to limited solubility and/or production in lower yields. Nonetheless, the calibration curves constructed from both sets of data followed similar trends.

Estimation of Detectable Oligomers. To estimate the maximum length of oligomers of glycine that we could reliably detect and analyze using this method, we collected an IP-HPLC chromatogram of a standard sample of low-molecular-weight (LMW) polyglycine (sold as molecular weight approx. 500-5,000 g/mol, but oligomers all the way down to free glycine were observed to be present). A saturated sample was prepared in $0.1 \%$ TFA in $\mathrm{H}_{2} \mathrm{O}$ and filtered before analysis by IP-HPLC. The chromatogram revealed resolved signals for all oligomers of Glyn up to Gly14 (Figure S19). While small peaks corresponding to Gly15 and Gly16 are also visible, we were not comfortable analyzing them due their small intensities and the start of a break in the pattern of retention times after Gly14. As a result, we report the estimated molar response factors for oligomers up to Gly14.

Statistical Treatment of Uncertainty and Error. Calibration curves for each of the six analytes for which we had standards (Gly1 though Gly6) appear in Figures S3-S8. The error bars 
for each point represent $95 \%$ confidence intervals based on three replicate trials (degrees of freedom $=2, t^{*}$ critical value $=4.303$ ). The slope of the least-squares regression line fitted to the plots of UV response vs. quantity of analyte is equal to the response factor, $f_{\mathrm{n}}$. The standard deviation of the slope, as determined by the LINEST function in Excel, was then used to calculate the $95 \%$ confidence interval for each response factor. ${ }^{3}$ These confidence intervals were determined using a $t^{*}$ critical value corresponding to $95 \%$ confidence based on five points in each plot (degrees of freedom $=3, t^{*}$ critical value $\left.=3.182\right)$.

Analysis of the Gly 8 Standard. We commissioned the synthesis of Gly from a commercial peptide vendor, but the sample obtained was not pure, so we did not incorporate Glys into our model for the extrapolation of molar response factors $\left(f_{\mathrm{n}}\right)$ of higher oligomers. We did subject the sample to the same analysis as Gly $1-6$ and determined $f_{8}=1940 \pm 15 \times 10^{15} \mathrm{~V}$. $\mathrm{sec} \cdot \mathrm{mol}^{-1}$. We expect this value to be low, as some of the mass of sample used to prepare the standard solution was that of impurities (not Gly8), and the molar response factor was based only on integrating the peak area for Gly8. In an attempt to correct the value of $f_{8}$, we integrated the peaks corresponding to the Gly6, Gly7, and Glyg impurities in Figure S1, and used the values of $f_{\mathrm{n}}$ reported in Table 1 for these oligomers to determine the moles present of these analytes in the impure Glys standard solution. We then subtracted the mass of these impurities from the mass of Gly8 used to prepare the standard solution. The corrected value for $f_{8}$ was determined to be 2143 $\times 10^{15} \mathrm{~V} \cdot \mathrm{sec} \cdot \mathrm{mol}^{-1}$, which compares favorably to $2101 \times 10^{15} \mathrm{~V} \cdot \mathrm{sec} \cdot \mathrm{mol}^{-1}$, the value for $f_{8}$ extrapolated using the regression line for Gly3-6. We do not report a confidence interval for $f_{8}$ due to the unclear uncertainty of our correction method. 


\section{Equations}

Equation S1. Determination of response factors.

$$
\begin{aligned}
& \text { Variables: } \quad \text { Gly } \quad=\text { peptide oligomer of } \mathrm{n} \text { glycine residues } \\
& m_{\mathrm{n}} \quad \text { = amount of injected Glyn analyte } \\
& \text { (units: mol) } \\
& A_{\mathrm{n}} \quad=\text { UV response of injected Glyn analyte } \\
& \text { (units: } \mathrm{mV} \cdot \mathrm{sec} \text { ) } \\
& f_{\mathrm{n}} \quad=\text { molar response factor for Glyn } \\
& \text { (units: } V \cdot \sec \cdot \mathrm{mol}^{-1} \text { ) } \\
& \text { UV response relations: } \quad A_{\mathrm{n}}=f_{\mathrm{n}} \times m_{\mathrm{n}} \\
& f_{\mathrm{n}}=A_{\mathrm{n}} / m_{\mathrm{n}}
\end{aligned}
$$

\begin{tabular}{|c|c|c|}
\hline Variables: & $\begin{array}{l}\bar{X} \\
t^{*} \\
\mathrm{~s} \\
\mathrm{n}\end{array}$ & $\begin{array}{l}=\text { sample mean } \\
=t \text {-distribution critical value } \\
=\text { sample standard deviation } \\
=\text { sample size }\end{array}$ \\
\hline
\end{tabular}

Equation S2. Determination of 95\% confidence intervals for UV response values.

Confidence Interval: $\quad 95 \% \mathrm{Cl}=\overline{\mathrm{X}} \pm t^{*} \frac{\mathrm{s}}{\sqrt{\mathrm{n}}}$ 


\section{Practical Notes}

Method Development. A mixed standard containing $\mathrm{Gly}_{2}, \mathrm{Gly}_{3}$, and $\mathrm{Gly}_{4}$ is a great checksample for the optimization or development of an IP-HPLC separation method for the analysis of oligomers of glycine. Gly3 and Gly4 are the most difficult oligomers to separate-if they are separating well, the separation method will likely work for all of the oligomers. We observed the resolution of Gly3 and Gly4 to worsen as the column aged. For instance, this difference in performance is observable between Figures 4 and S10 (fresh column) and Figure S19 (column near the end of its life).

pH of Mobile Phase. It is important to ensure that the $\mathrm{pH}$ of the mobile phase is consistent from one batch to another. Not only will the $\mathrm{pH}$ affect the retention times of the analytes, it could also have an impact on their extinction coefficients, potentially reducing the accuracy of the measurements. Our mobile phase was adjusted to $\mathrm{pH} 2.5$ with HPLC-grade $\mathrm{H}_{3} \mathrm{PO}_{4}$ in $2 \mathrm{~L}$ batches using a Jenway $3510 \mathrm{pH}$ Meter.

Limits of Quantitation. We report limit of quantitation (LOQ) values for Gly2 through Gly6 in Table S4. These LOQ values were determined by taking the sample standard deviation of the three measured UV responses (peak areas in the chromatogram) for the lowest concentration of Gly2-6 used to prepare each of the calibration curves in Figures S4-S8, then multiplying by 10 and dividing by the slope of the calibration curve.

Control Experiment for Interference by DKP. Cyclic diglycine (2,5-diketopiperazine, DKP) hydrolyzes to form linear diglycine (Gly2). As we do not quantify DKP but do report yields of Gly2, we ran a control experiment to test whether the DKP present in a product mixture could hydrolyze during analysis to appear as Gly2 and thus inflate its yield. We prepared a 70 $\mathrm{mM}$ solution of DKP in the $0.1 \%$ trifluoroacetic acid solution used to prepare samples for 
analysis and let the sample stand for 5 hours to simulate the maximum time a sample would wait prior to injection on the HPLC instrument once prepared. After 5 hours, the peak corresponding to Gly2 was below the limit of quantitation (LOQ) for Gly2 reported in Table S4, suggesting the interference of DKP in our analysis is insignificant. 
Figure S1. IP-HPLC chromatogram for the commercial sample of Gly8. The inset chromatogram depicts a magnification of the parent chromatogram, with the peaks for Gly8 aligned. Note the impurities present from other oligomers of glycine, including Gly9. Due to the lack of purity of this sample, we did not incorporate data collected on Glys into the extrapolation of the higher oligomers of Glyn.

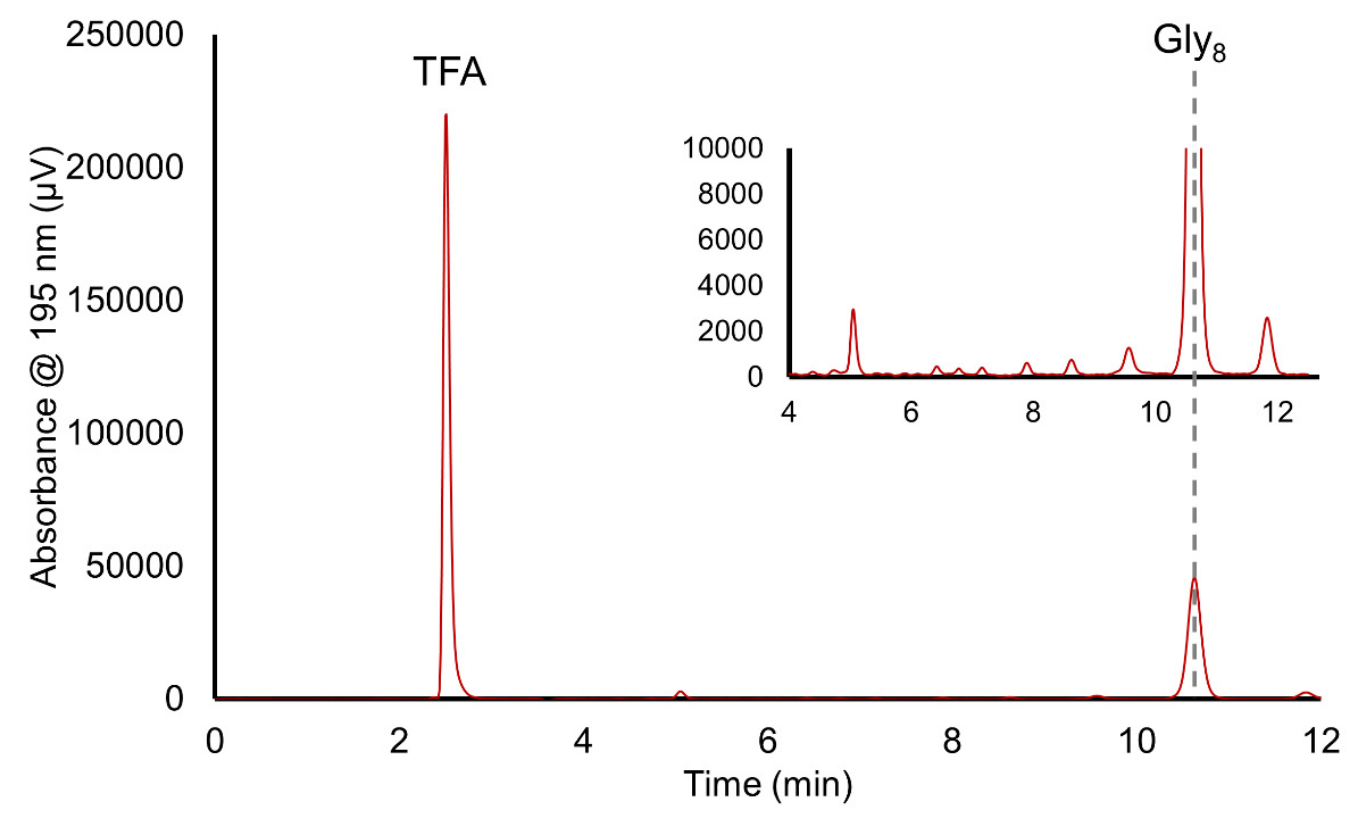


Figure S2. Overlaid chromatograms of Gly4 standards subjected to analysis by IP-HPLC. The peaks for each run were integrated to determine the UV responses, which were then plotted against the moles of analyte injected into the instrument to determine the molar response factor $f_{4}$ (see Figure S6). Identical experiments were conducted for each Glyn standard to determine values for $f_{1-6,8}$ (see Figures S3-S9). Concentrations of [Gly4] $=0.290 \mathrm{mM}$ (violet), $0.725 \mathrm{mM}$ (blue), $1.45 \mathrm{mM}$ (green), $2.17 \mathrm{mM}$ (yellow), $2.90 \mathrm{mM}$ (red).

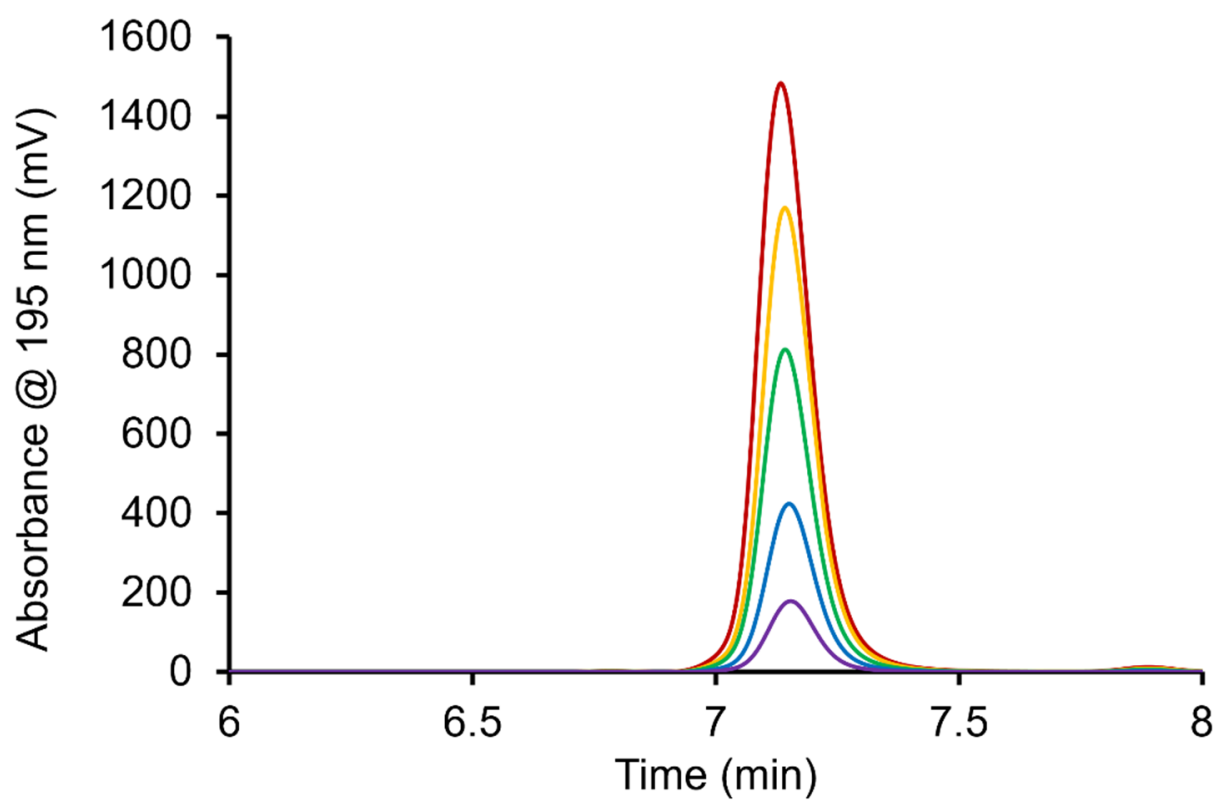


Figure S3. The calibration curve of glycine. Error bars represent $95 \%$ confidence intervals based on three measurements at each concentration. The slope is equal to the molar response factor $f_{1}$ at $195 \mathrm{~nm}$ and is reported as a 95\% confidence interval determined by the statistical analysis reported in the Supplemental Experimental section of the SI.

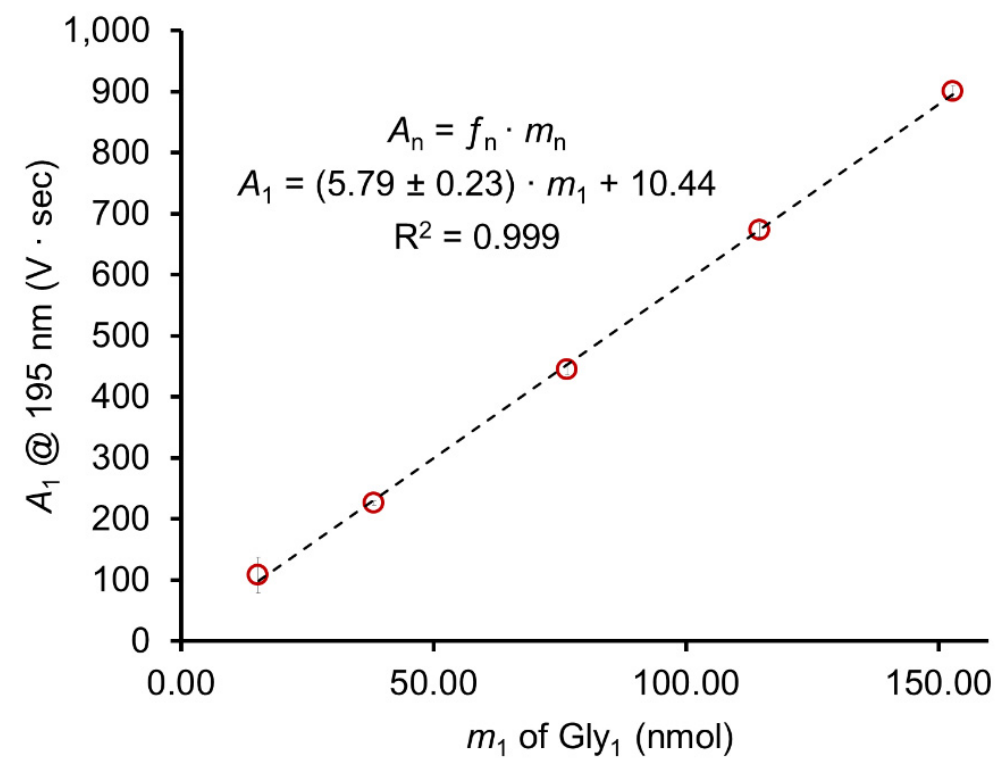

Figure S4. The calibration curve of Gly2. Error bars represent 95\% confidence intervals based on three measurements at each concentration. The slope is equal to the molar response factor $f_{2}$ at $195 \mathrm{~nm}$ and is reported as a 95\% confidence interval determined by the statistical analysis reported in the Supplemental Experimental section of the SI.

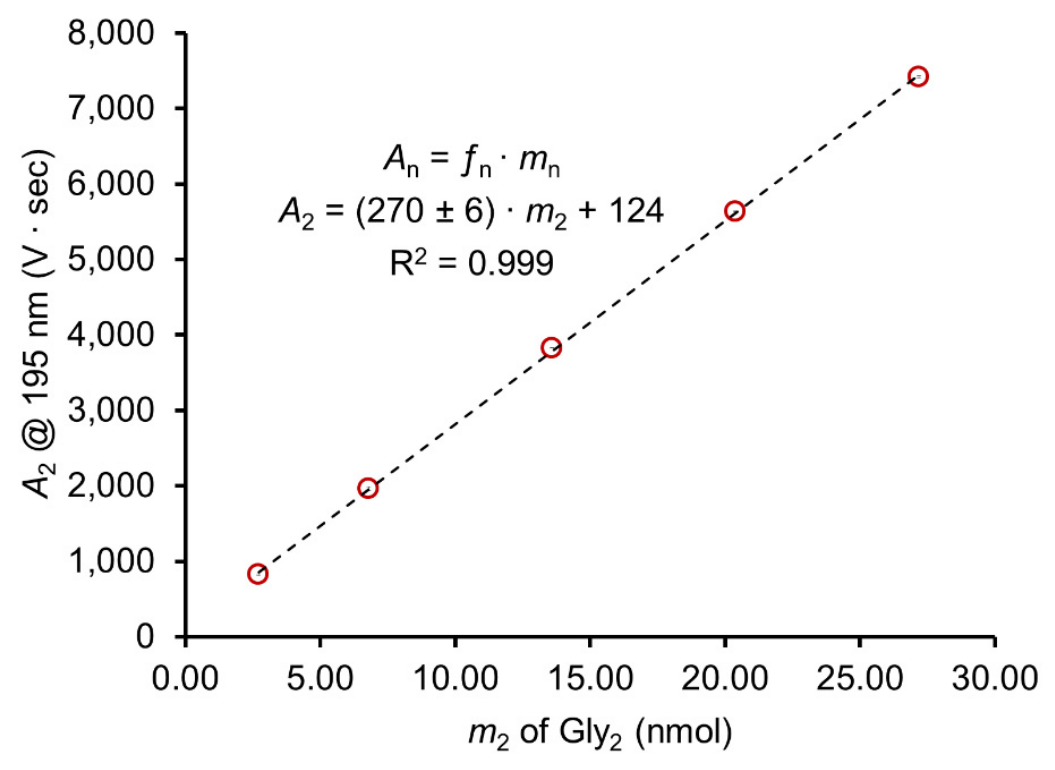


Figure S5. The calibration curve of Gly3. Error bars represent 95\% confidence intervals based on three measurements at each concentration. The slope is equal to the molar response factor $f_{3}$ at $195 \mathrm{~nm}$ and is reported as a 95\% confidence interval determined by the statistical analysis reported in the Supplemental Experimental section of the SI.

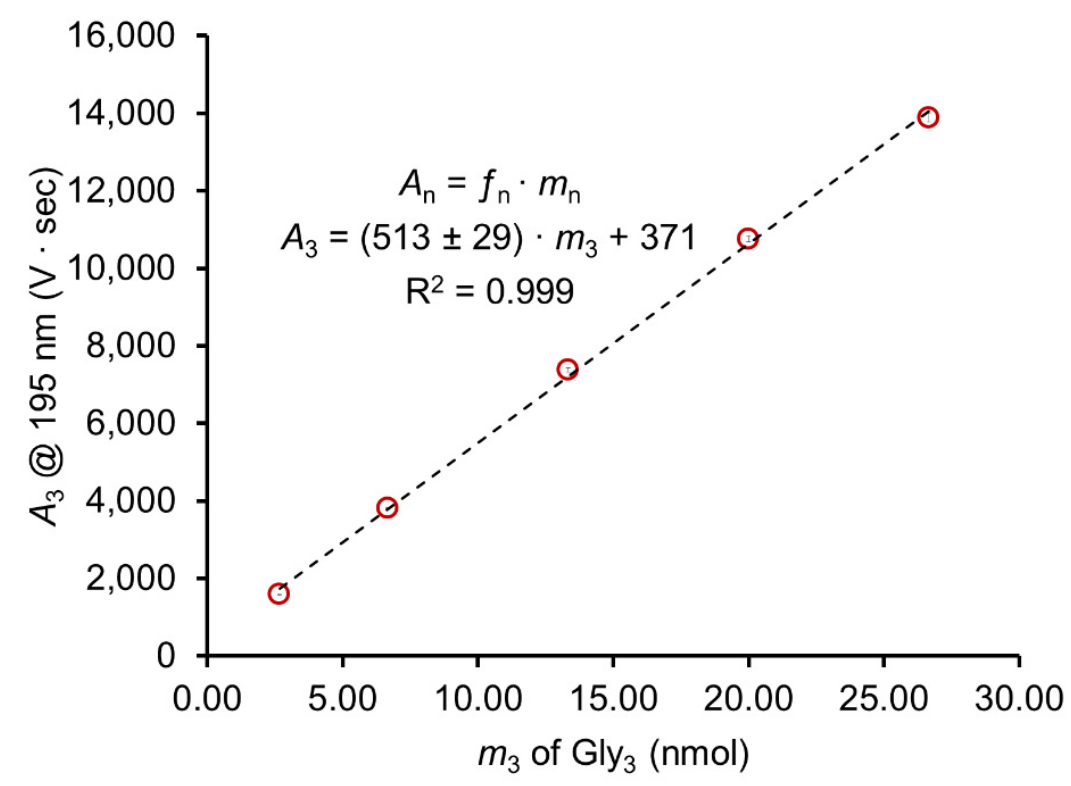

Figure S6. The calibration curve of Gly4. Error bars represent 95\% confidence intervals based on three measurements at each concentration. The slope is equal to the molar response factor $f_{4}$ at $195 \mathrm{~nm}$ and is reported as a 95\% confidence interval determined by the statistical analysis reported in the Supplemental Experimental section of the SI. An identical plot appears in the main paper as Figure 2b, where it represents a typical calibration curve.

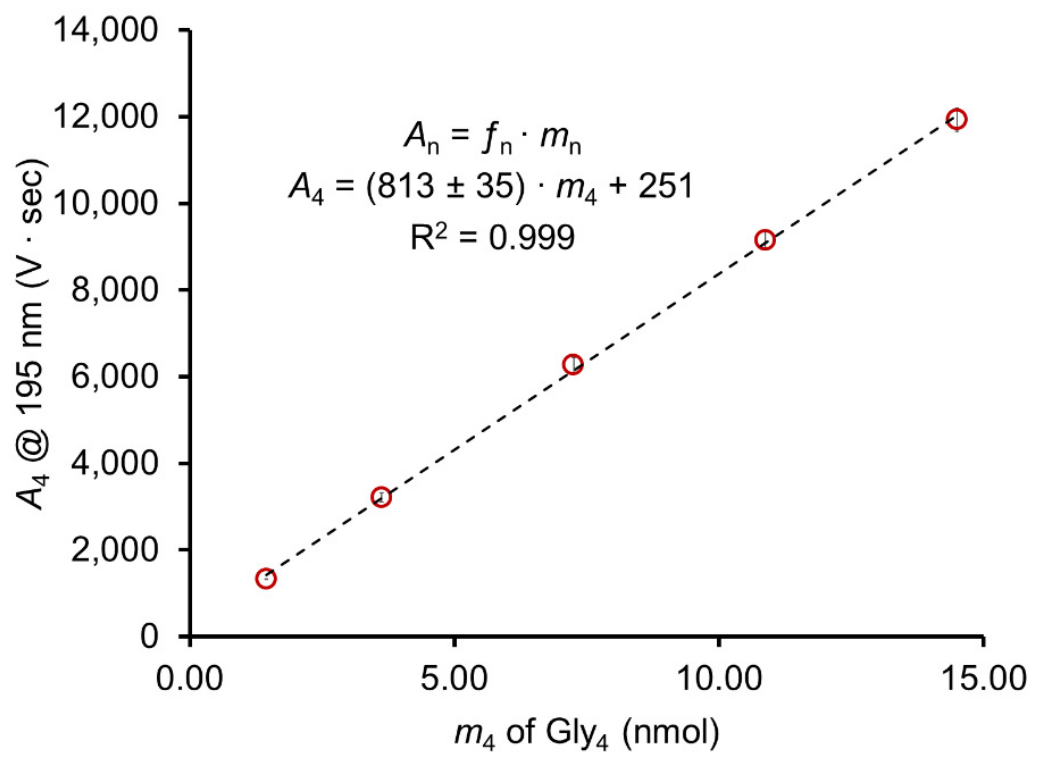


Figure S7. The calibration curve of Gly5. Error bars represent 95\% confidence intervals based on three measurements at each concentration. The slope is equal to the molar response factor $f_{5}$ at $195 \mathrm{~nm}$ and is reported as a 95\% confidence interval determined by the statistical analysis reported in the Supplemental Experimental section of the SI.

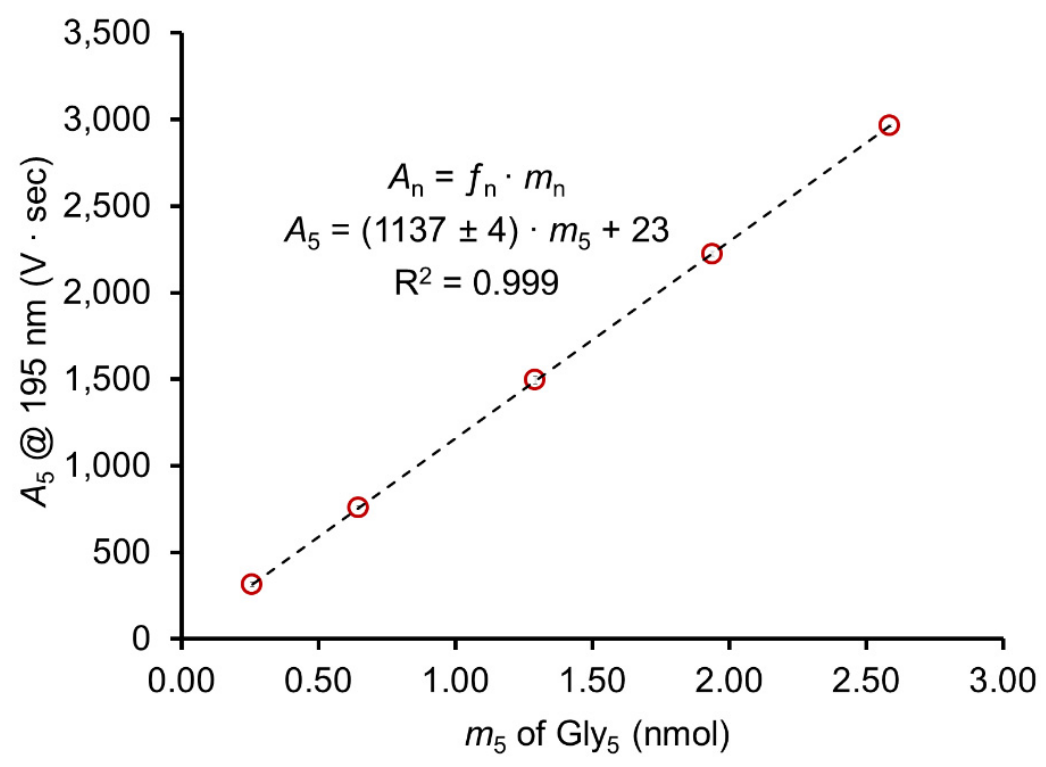

Figure S8. The calibration curve of Gly6. Error bars represent $95 \%$ confidence intervals based on three measurements at each concentration. The slope is equal to the molar response factor $f_{6}$ at $195 \mathrm{~nm}$ and is reported as a $95 \%$ confidence interval determined by the statistical analysis reported in the Supplemental Experimental section of the SI.

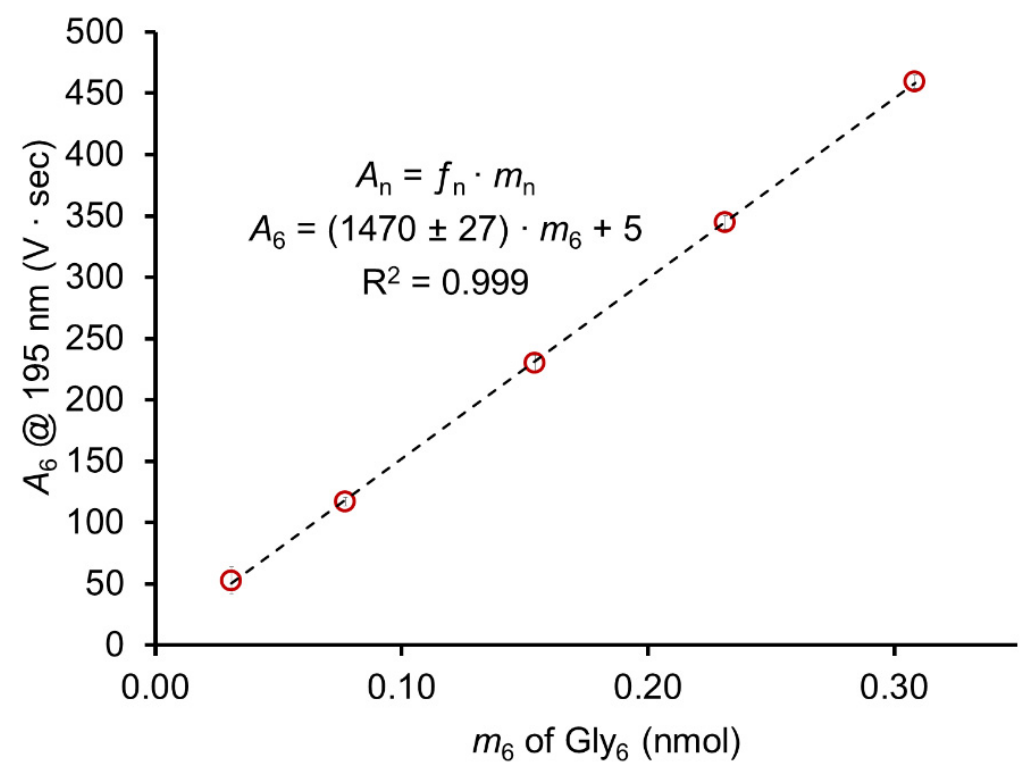


Figure S9. The calibration curve of Gly8. Error bars represent 95\% confidence intervals based on three measurements at each concentration. The slope is equal to the molar response factor $f_{8}$ at $195 \mathrm{~nm}$ and is reported as a 95\% confidence interval determined by the statistical analysis reported in the Supplemental Experimental section of the SI. This value for $f_{8}$ is uncorrected in the sense that this analysis did not account for the fact that the sample contained impurities of other Glyn compounds, up to Gly9. A corrected value of $f_{8}=2143 \times 10^{15} \mathrm{~V} \cdot \mathrm{sec} \cdot \mathrm{mol}^{-1}$ was determined by a method described on page S5.

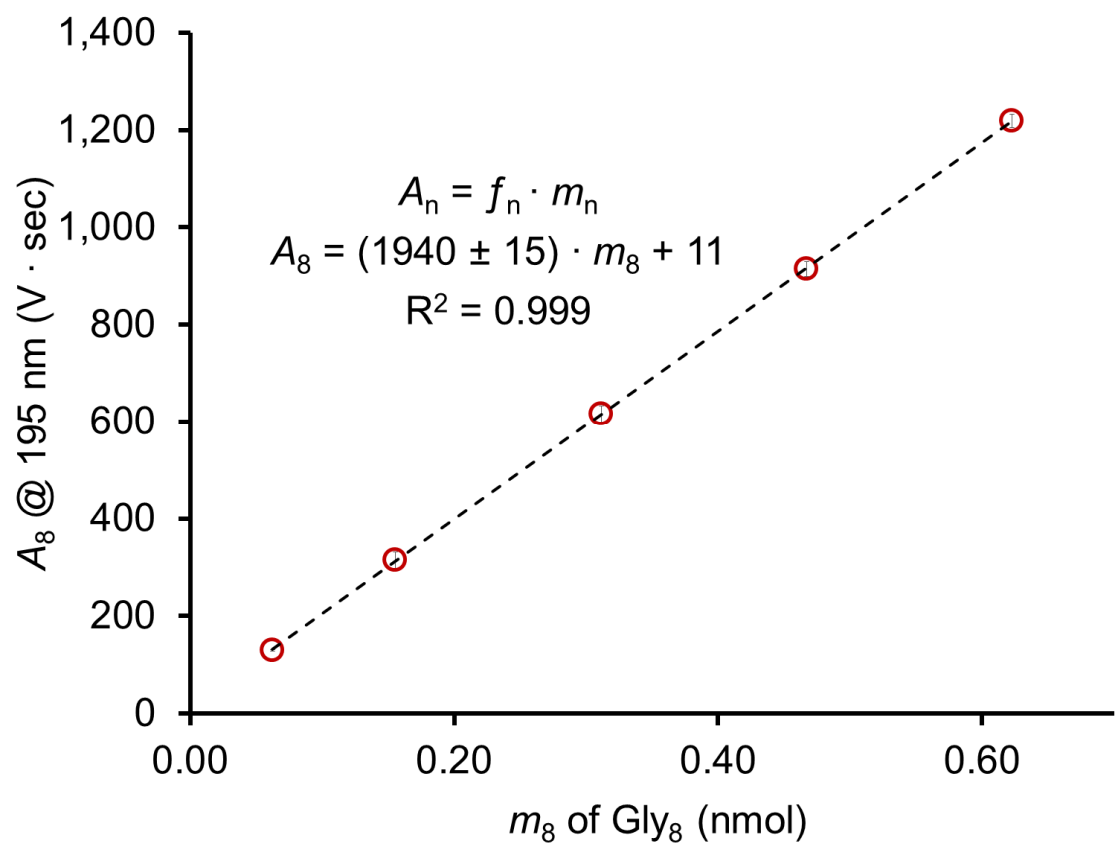


Figure S10. IP-HPLC chromatogram of a mixed standard of Gly1-6.

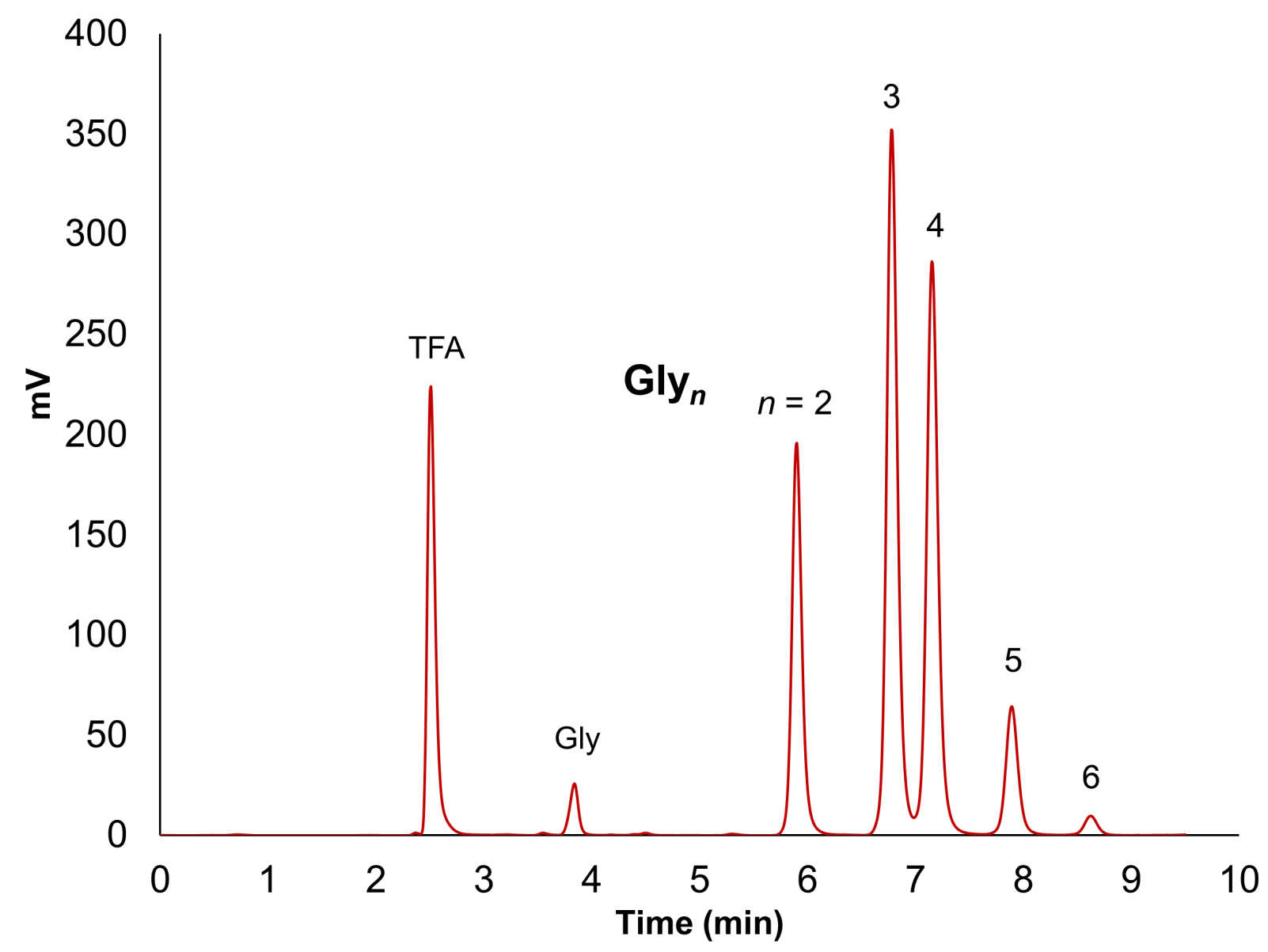

Table S1. Approximate retention times of standards of oligomers of glycine for the reported IP-HPLC method.

\begin{tabular}{lc}
\hline \multicolumn{1}{c}{ Standard } & Retention Time (min) \\
\hline Glycine (Gly) & 3.8 \\
Gly-Gly (Gly2) & 5.9 \\
Gly-Gly-Gly (Glys) & 6.7 \\
Gly-Gly-Gly-Gly (Gly4) & 7.1 \\
Gly-Gly-Gly-Gly-Gly (Gly5) & 7.9 \\
Gly-Gly-Gly-Gly-Gly-Gly (Gly6) & 8.6 \\
Gly-Gly-Gly-Gly-Gly-Gly-Gly-Gly (Glys) & 10.9 \\
\hline
\end{tabular}


Figure S11. An ${ }^{1} \mathrm{H}$ NMR spectrum of standard solution of glycine, including $2.5 \mathrm{mM}$ tertbutanol to verify the concentration of the standard solution.

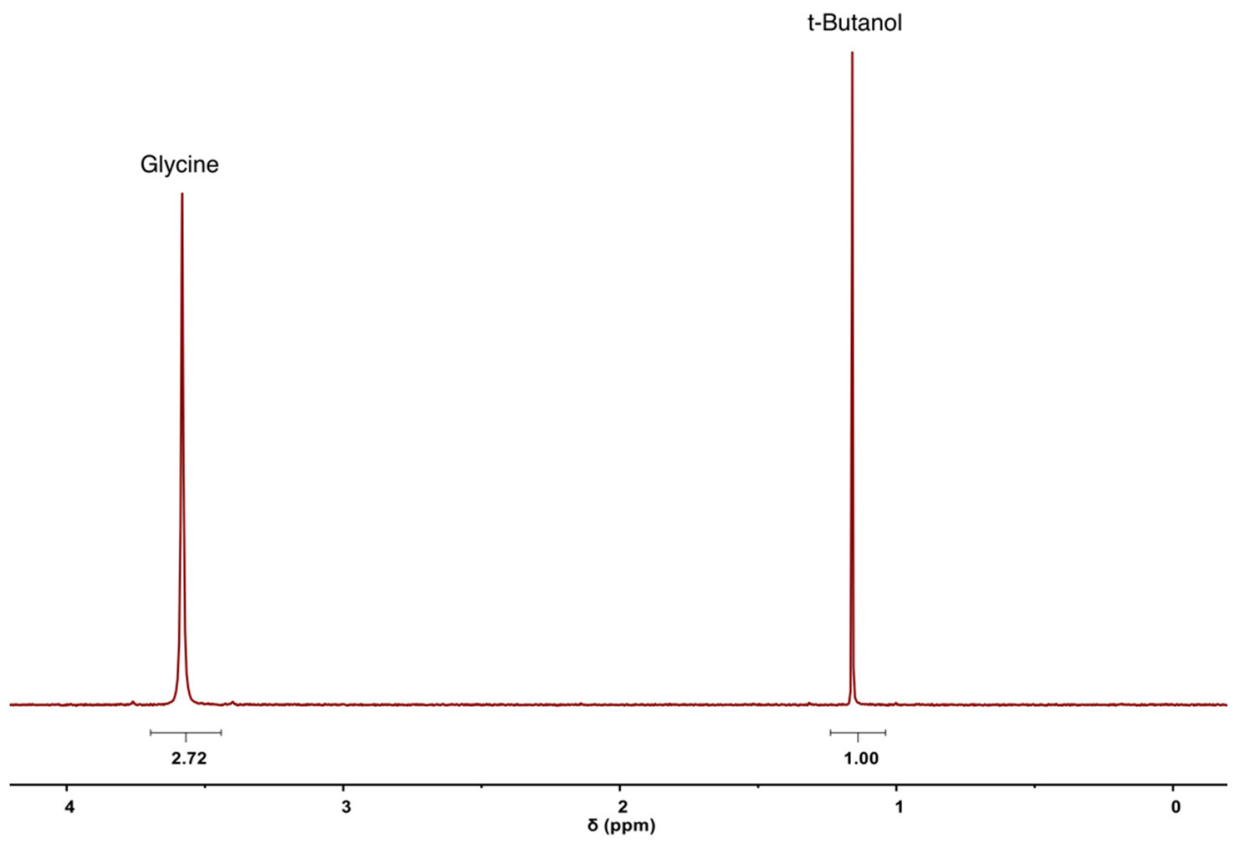

Figure S12. An ${ }^{1} \mathrm{H}$ NMR spectrum of standard solution of Gly2, including $2.5 \mathrm{mM}$ tert-butanol to verify the concentration of the standard solution.

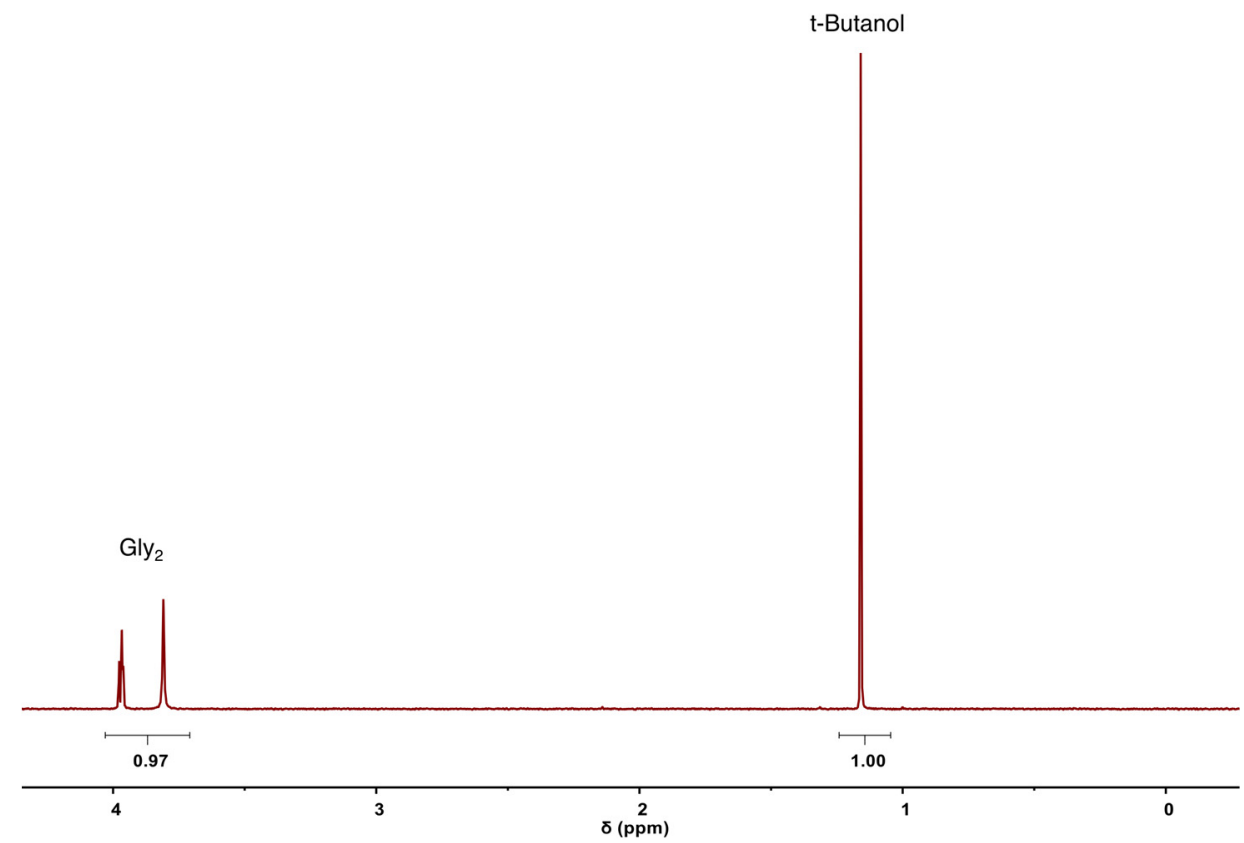


Figure S13. An ${ }^{1} \mathrm{H}$ NMR spectrum of standard solution of Gly3, including $2.5 \mathrm{mM}$ tert-butanol to verify the concentration of the standard solution.

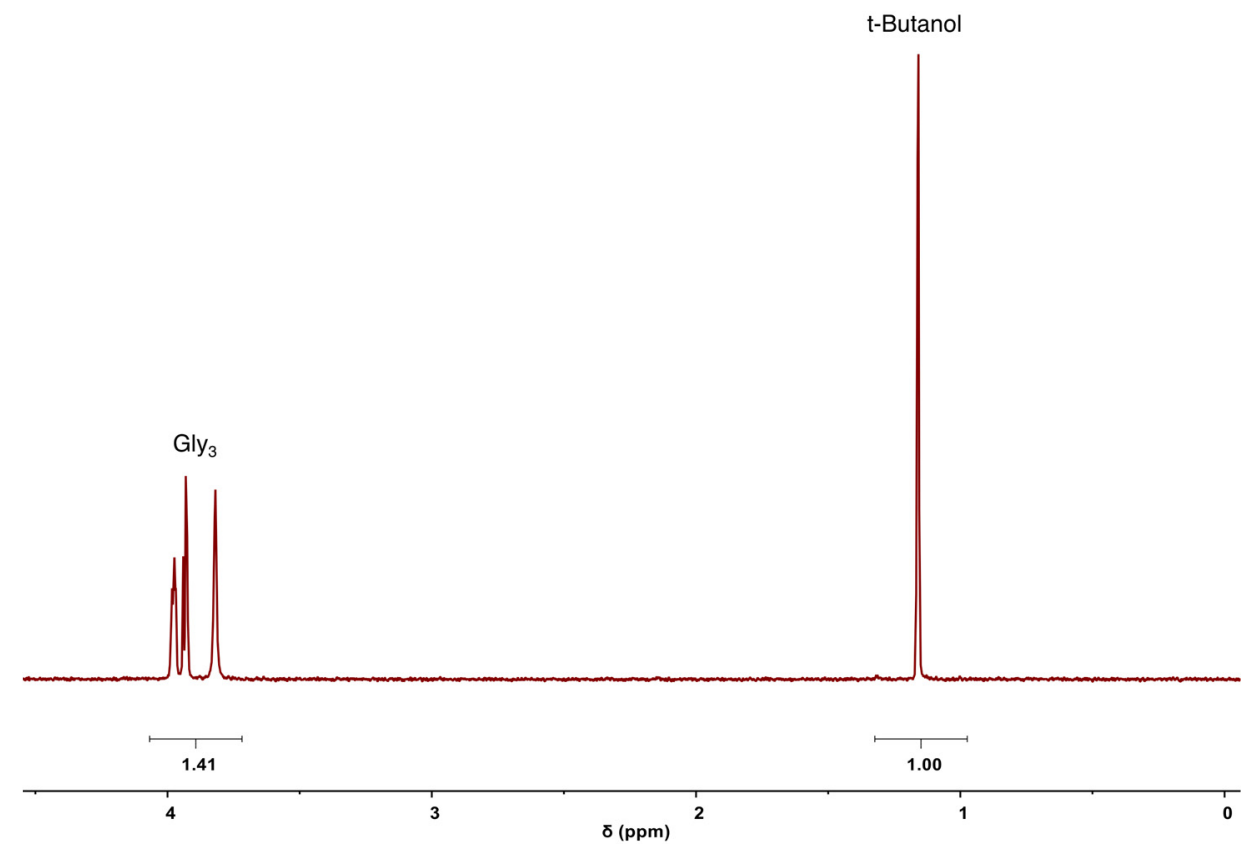

Figure S14. An ${ }^{1} \mathrm{H}$ NMR spectrum of standard solution of Gly4, including $2.5 \mathrm{mM}$ tert-butanol to verify the concentration of the standard solution.

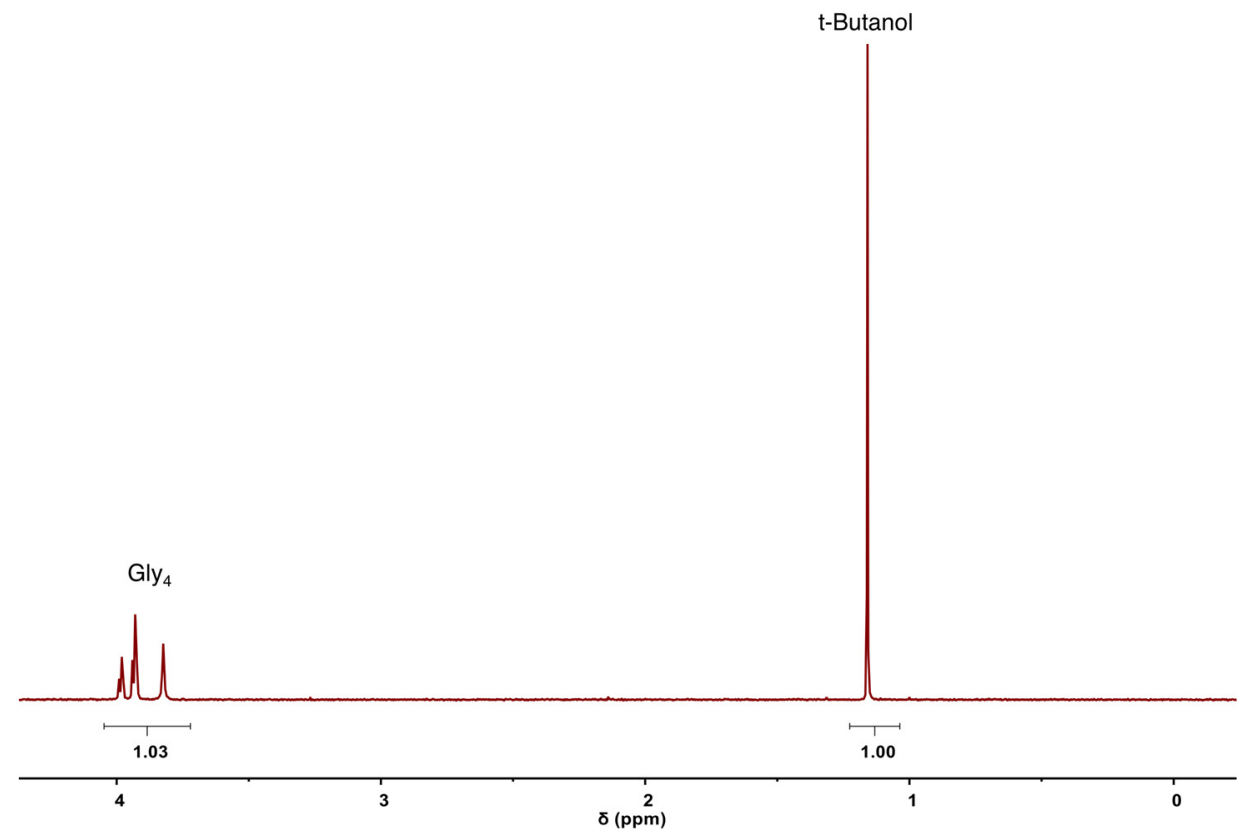


Figure S15. An ${ }^{1} \mathrm{H}$ NMR spectrum of standard solution of Gly5, including $2.5 \mathrm{mM}$ tert-butanol to verify the concentration of the standard solution.

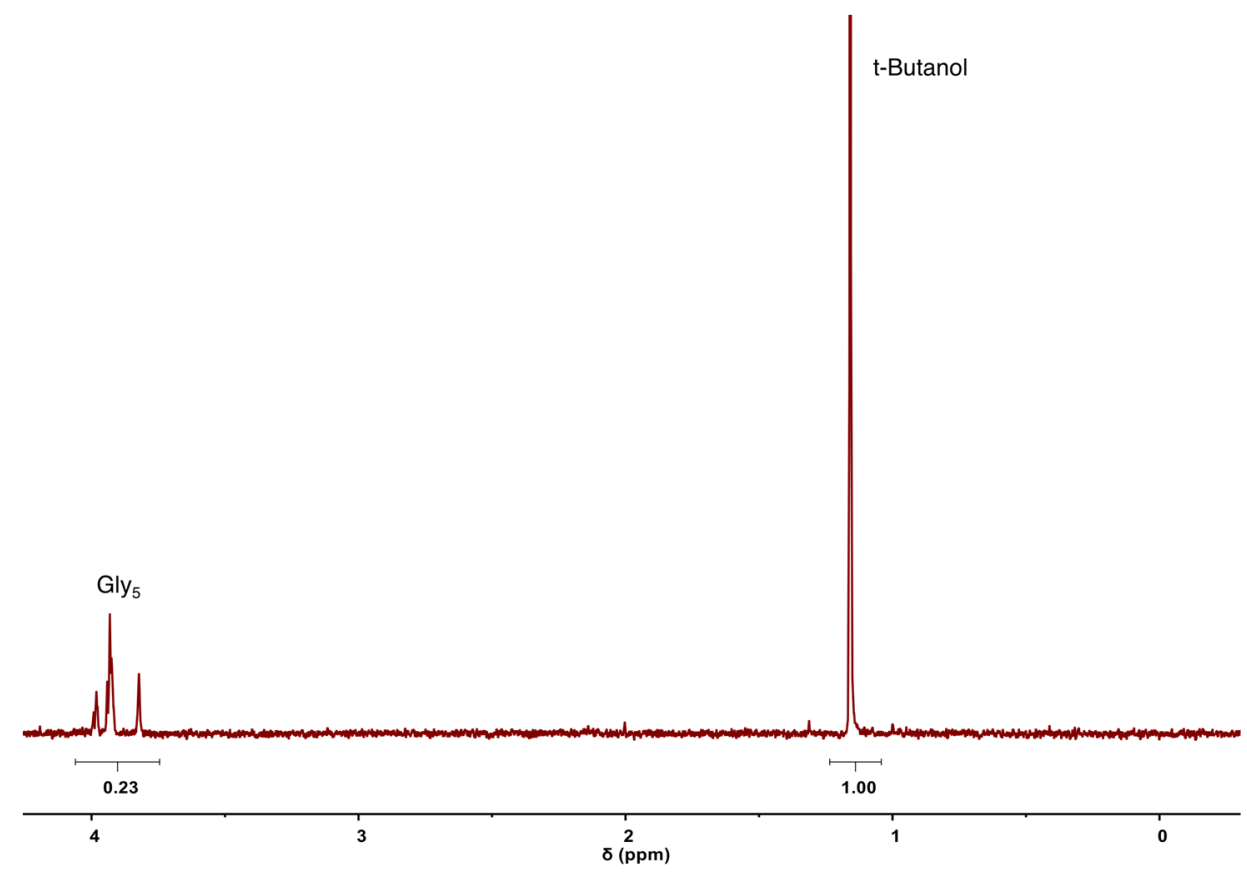

Figure S16. An ${ }^{1} \mathrm{H}$ NMR spectrum of standard solution of Gly6, including $2.5 \mathrm{mM}$ tert-butanol to verify the concentration of the standard solution.

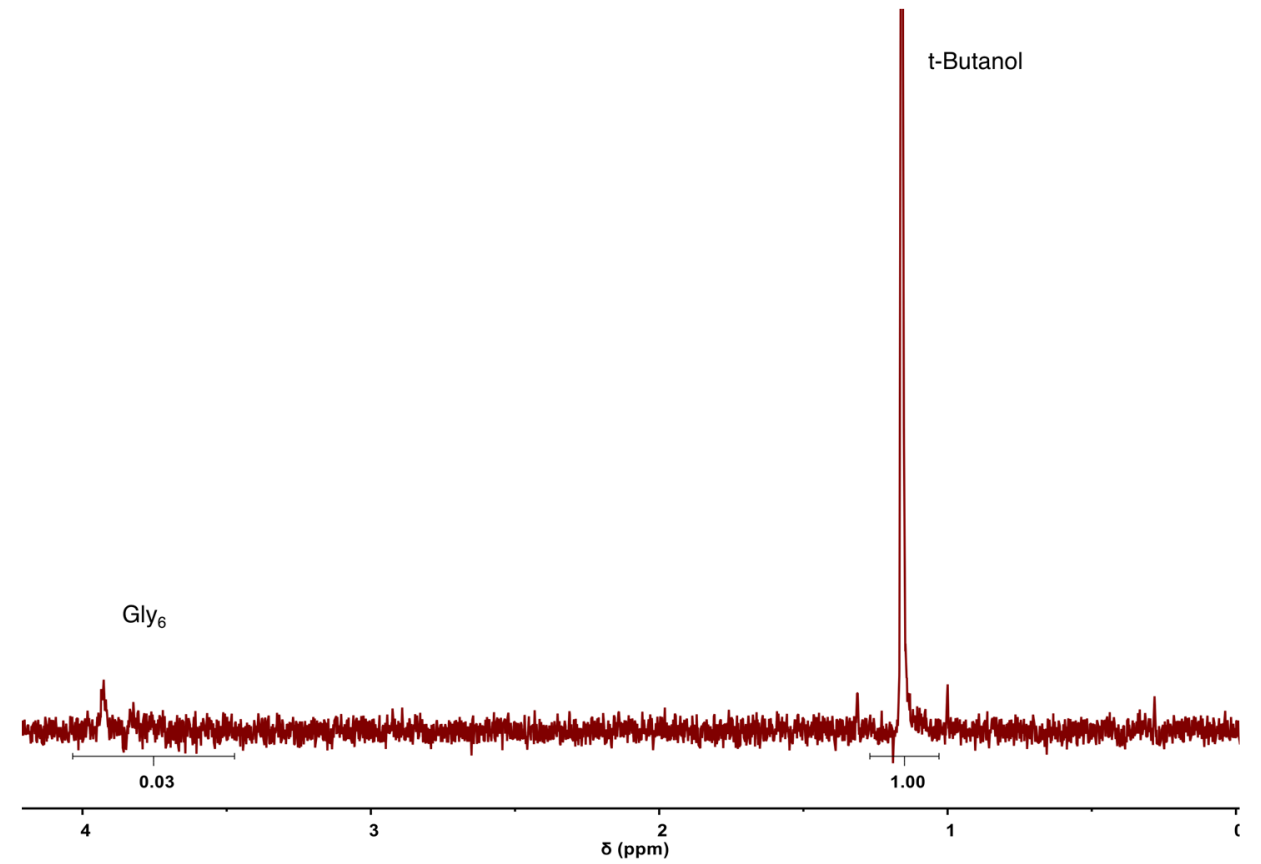


Figure S17. An ${ }^{1} \mathrm{H}$ NMR spectrum of mixed standards of Gly1-6, including $2.5 \mathrm{mM}$ tert-butanol as an internal standard.

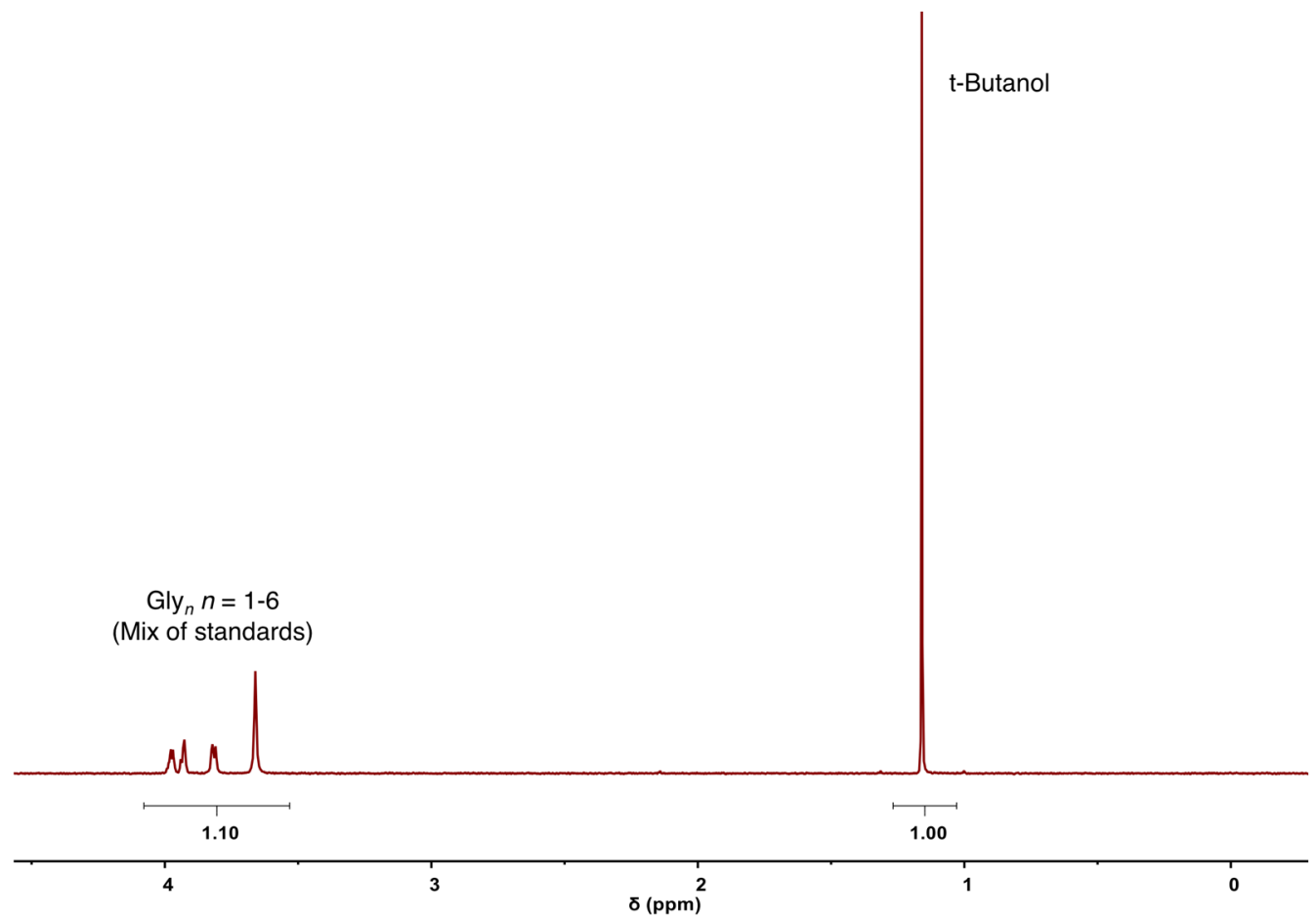


Figure S18. UV-vis spectra of the IP running buffer (blue curve, where a background spectrum of milli-Q has been subtracted) and a solution of Gly4 standard (red curve, where a background spectrum of the IP running buffer has been subtracted).

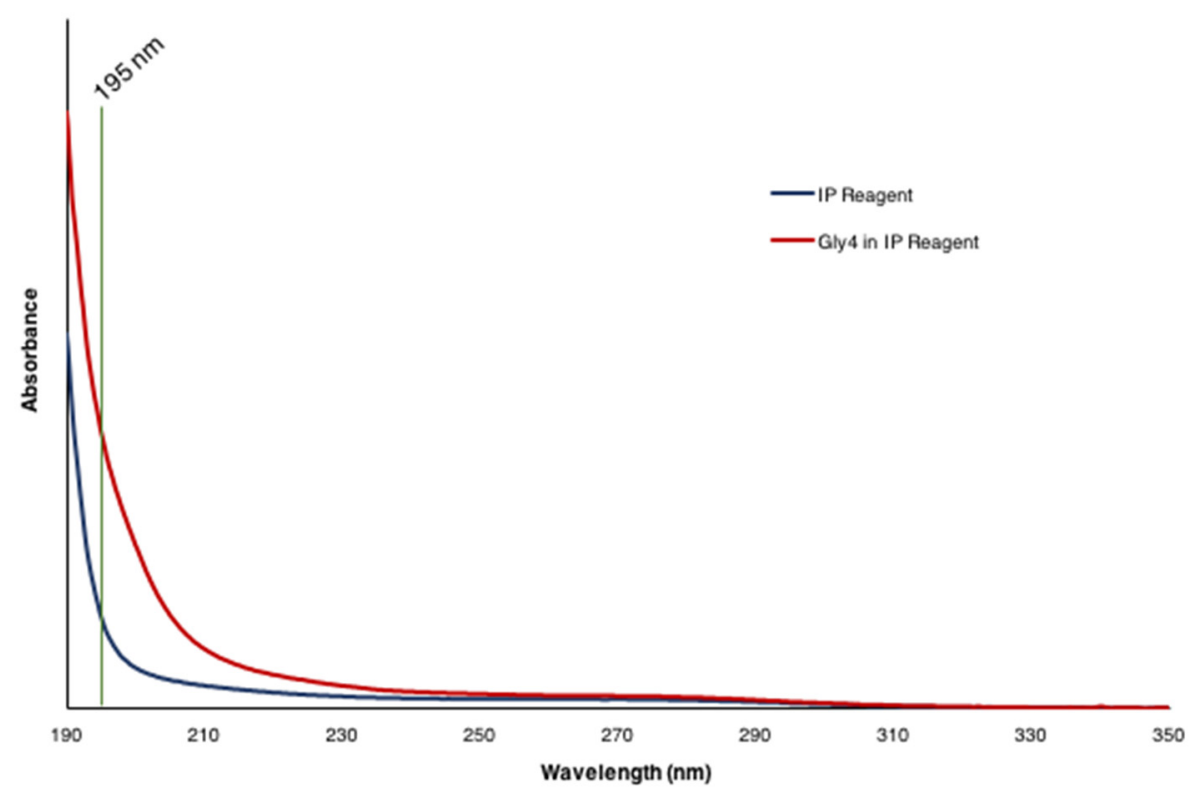


Table S2. Measured UV responses (peak areas, in units of $\mathrm{mV} \cdot \mathrm{sec}$ ) for each oligomer in a mixed standard of Gly $1-6$ and the resulting concentration of total Glyn. Each column corresponds to the data collected from one of three trials. A sample chromatogram from one trial appears as Figure S10.

\begin{tabular}{|c|c|c|c|}
\hline Analyte & 1 & 2 & 3 \\
\hline Glycine & 151232 & 151025 & 148850 \\
\hline Gly2 & 1296964 & 1298317 & 1298970 \\
\hline $\mathrm{Gly}_{3}$ & 2528292 & 2534156 & 2532483 \\
\hline Gly4 & 2186451 & 2187102 & 2185488 \\
\hline Gly5 & 532189 & 531497 & 530960 \\
\hline Gly 6 & 81008 & 82908 & 80612 \\
\hline Total Gly $_{n}(\mathrm{mM})$ & 12.79 & 12.79 & 12.71 \\
\hline Average $\pm 95 \%$ CI & \multicolumn{3}{|c|}{$12.76 \% \pm 0.11 \%$} \\
\hline Total Gly by NMR (mM) & \multicolumn{3}{|c|}{12.35} \\
\hline Percent Difference & \multicolumn{3}{|c|}{$3.2 \%$} \\
\hline
\end{tabular}

Table S3. Measured UV responses (peak areas, in units of $\mathrm{mV} \cdot \mathrm{sec}$ ) of each Glyn oligomer in the product mixtures of three replicate trials of the oligomerization of glycine. A sample chromatogram from one trial appears as Figure 4 of the main paper.

\begin{tabular}{|c|c|c|c|}
\hline Analyte & 1 & 2 & 3 \\
\hline Glycine & N/A & N/A & $\mathrm{N} / \mathrm{A}$ \\
\hline Gly2 & 10299513 & 10218977 & 10022841 \\
\hline $\mathrm{Gly}_{3}$ & 12161779 & 12545225 & 11981873 \\
\hline $\mathrm{Gly}_{4}$ & 6590787 & 6684185 & 6465976 \\
\hline Gly5 & 3946077 & 4157463 & 3936857 \\
\hline Gly 6 & 2021211 & 2122018 & 2037391 \\
\hline Gly7 & 1415521 & 1423520 & 1432869 \\
\hline Gly 8 & 674667 & 705527 & 696777 \\
\hline Gly9 & 360496 & 377628 & 361111 \\
\hline $\mathrm{Gly}_{10}$ & 438106 & 369598 & 425484 \\
\hline Total Yield (\%) & 49.52 & $\mathbf{5 0 . 3 0}$ & 48.70 \\
\hline Average $\pm 95 \%$ CI & \multicolumn{3}{|c|}{$49.5 \% \pm 2.0 \%$} \\
\hline
\end{tabular}


Figure S19. An IP-HPLC chromatogram of an unmodified sample of low-molecular-weight polyglycine. The detection of up to Gly 14 establishes the upper limit for analysis of oligomers of glycine using this method.

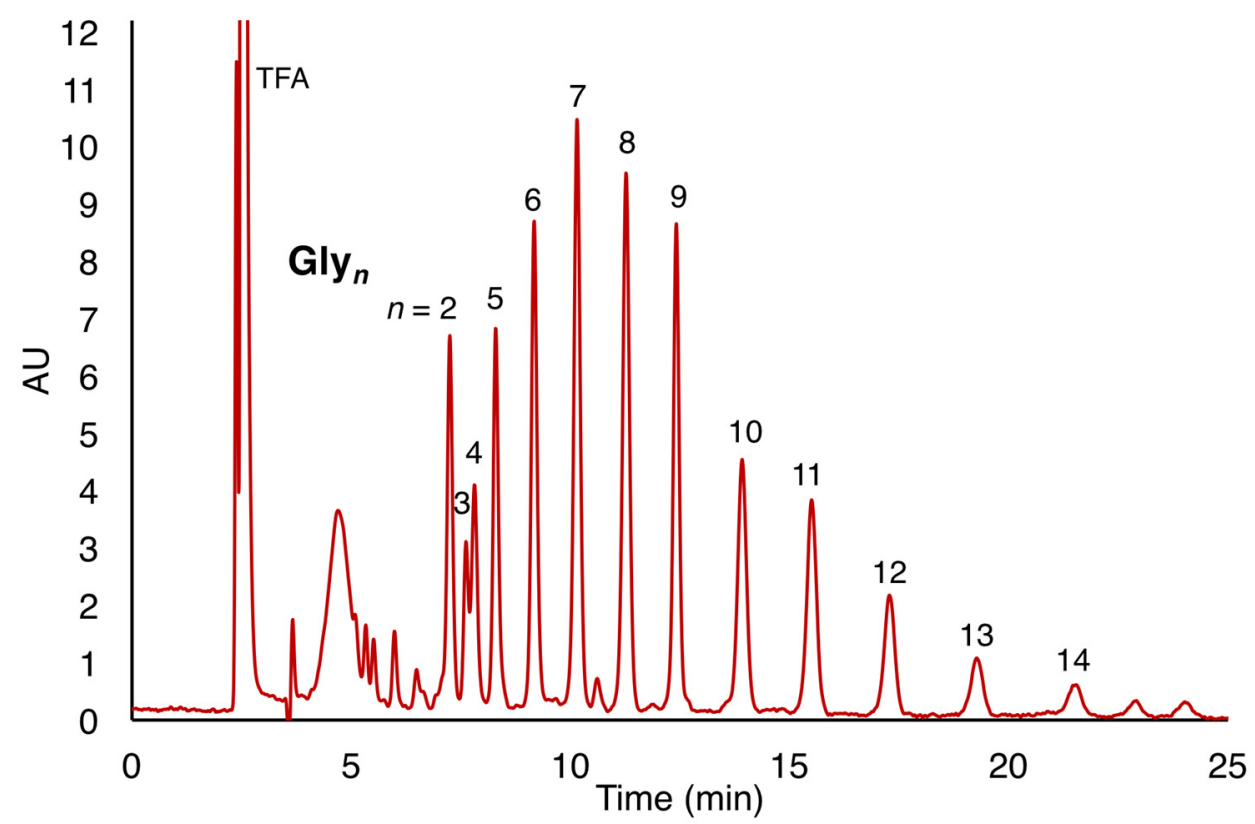


Table S4. Measured limits of quantitation (LOQ) for Gly2 through Gly6 per injection for the described IP-HPLC method.

\begin{tabular}{cr}
\hline Analyte & LOQ (nmol) \\
\hline \hline Gly2 & 0.203 \\
Gly3 & 0.134 \\
Gly4 & 0.053 \\
Gly5 & 0.048 \\
Gly6 & 0.031 \\
\hline
\end{tabular}




\section{Supplemental References}

1. Pauli, G. F.; Jaki, B. U.; Lankin, D. C., A routine experimental protocol for qHNMR illustrated with taxol. J. Nat. Prod. 2007, 70, 589-595.

2. Campbell, T. D.; Hart, C. A.; Febrian, R.; Cheneler, M. L.; Bracher, P. J., The opposite effect of $\mathrm{K}^{+}$and $\mathrm{Na}^{+}$on the hydrolysis of linear and cyclic dipeptides. Tetrahedron Lett. 2018, 59, 2264-2267.

3. Morrison, F. A. Obtaining Uncertainty Measures on Slope and Intercept of a Least Squares Fit with Excel's LINEST, http://pages.mtu.edu/ fmorriso/cm3215/UncertaintySlopeInterceptOfLeastSquaresFit.pdf. (accessed 22 August 2018). 